\title{
Innate Talent in Sport: Beware of an organismic asymmetry - comment on Baker \& Wattie
}

\author{
Keith Davids ${ }^{1, *}$ \& Duarte Araújo ${ }^{2}$ \\ 1 Centre for Sports Engineering Research, Sheffield Hallam University, UK \\ 2 CIPER, Faculdade de Motricidade Humana, Universidade de Lisboa, Lisbon, Portugal \\ * Corresponding author: Centre for Sports Engineering Research, Sheffield Hallam University, 11 Broomgrove Road, Sheffield S10 2LX, UK \\ Tel: +44 (0)114225 2255 \\ E-Mail: k.davids@shu.acu.uk
}

\section{COMMENTARY}

\section{Article History:}

Submitted $5^{\text {th }}$ January 2019

Accepted $1^{\text {st }}$ February 2019

Published $7^{\text {th }}$ May 2019

\section{Handling Editor:}

Lisa Steidl-Müller

University of Innsbruck, Austria

Editor-in-Chief:

Martin Kopp

University of Innsbruck, Austria

\begin{abstract}
Of fundamental, theoretical and practical, relevance to sport science is the conceptualisation of talent, skill and expertise. Revisiting the question of innate talent is timely, given current pressure on young children selected to specialise in sport at an early age. Here, we re-iterate the conceptualisation of talent, skill and expertise in sport as an increasingly functional relationship between an athlete and a specific performance environment, developed over the macro-timescale of years and decades. This ecological dynamics rationale avoids an organismic asymmetry, the bias towards explanations of human behaviour which over-emphasise the role of inherent properties and qualities, identifiable through early selection. An ecological dynamics rationale, eschewing the organismic asymmetry of innate talent, in favour of exploiting and developing individual functionality in specific performance environments, challenges coaches and sport scientists to collaborate in new models for developing talent, skill and expertise.
\end{abstract}

Keywords:

Talent development - skill adaptation - ecological dynamics - organismic asymmetry- personenvironment interactions

Citation:

Davids, K. \& Araújo, D. (2019): Innate Talent in Sport: Beware of an organismic asymmetry - comment on Baker \& Wattie Current Issues in Sport Science, 4:102. doi: 10.15203/CISS_2019.102

This is a commentary on a CISS report article authored by Baker, J. \& Wattie, N. (2018). Innate talent in sport: Separating myth from reality. Current Issues in Sport Science, 3:006. doi: 10.15203/CISS_2018.006

\section{Introduction}

Baker and Wattie's re-examination of the concept of innate talent promotes a useful re-evaluation of this original proposition in psychology, but particularly drew our attention to two key, take-home messages: (i) their conclusion that the concept of innate talent had some validity, and (ii), that it also had limited practical utility for sports organisations.

Here, we discuss how conceptualisation of talent from an ecological dynamics perspective is not aligned with the first of Baker and Wattie's messages. The conceptualisation of 'talent' as innate does, however, raise significant questions about other human characteristics, and more practically, about how it can be utilized by sports organizations to develop athletes. This is not a trivial matter, as noted by Baker and Wattie, since currently much time and effort in system development and practitioner support is devoted in organized sport for early identification and selection of 'talented' children predicted to succeed at the highest performance levels.

Previously, we proposed, utilising the conceptualization of ecological dynamics and empirical data, how copious financial support and time involved in talent identification and early selection is misplaced due to a fundamental organismic asymmetry or biased explanation for behaviour centred on internal entities 
(Davids \& Araújo, 2010). The inherent nonlinearity of learning, development, and expertise in sport, strikingly highlights the misconceptions of a priori talent identification. Conceptualization of humans as complex adaptive systems, with nonlinear trajectories observed at different timescales (e.g., growth and maturation, development and learning), raises important questions, including: Why would measuring a putative innate property at birth or in the foetus be relevant for predicting later sport performance? Such properties, measured at those specific points in time, could be considered innate, but will certainly change over time due to interacting constraints of gene interactions, epigenetics, experiences, and chance. Moreover, whichever property (e.g., genes, molecular composition or hormonal markers) is measured after conception could never be considered as innate with certainty, given that development and environmental constraints are already influencing it and its relevance. Instead, these questions signal the supremacy of human adaptation and learning emanating from continuous individual-environment interactions (Araújo \& Davids, 2011).

Talent development is predicated on functionality of individualenvironment interactions

Due to centrality of individual-environment interactions in human behaviour, an ecological dynamics rationale places a greater emphasis on talent development in sport, defining talent, wrapped up with skill and expertise, not as innate qualities which individuals either have or do not have, but rather as: an increasingly functional relationship developed between a performer and a specific performance environment over macrotimescales (Davids, Güllich, Araújo \& Shuttleworth, 2017).

What does a functional relationship with a competitive performance environment in sport look like? Of fundamental importance is an athletess capacity to attain intended task goals during performance, predicated on consistently achieving performance outcomes and results, by satisfying constraints of ever-changing competitive environments. Functional behaviours emerge in competitive performance environments and relevant performance solutions may vary over different timescales for athletes, including: (i) within individuals during performance, and athletic development (through motor learning, changes in effectivities - capacities and skills -, adaptations to training and conditioning or through growth and maturation); and (ii), over the macro-timescale of years and decades as sports evolve, constrained by innovations in tactical trends, sport formats, playing surfaces, rules and regulations, and updated equipment and technology. These changes shape sports over time and are rarely predictable, illustrating the futility of defining innate talent in young children.

An athlete's capacity to functionally interact with varied and dynamic performance constraints is predicated on dexterity (adaptive flexibility). Insights on dexterity were provided by Nikolai Bernstein, the Russian physiologist who has had an enormous impact on theoretical development in the human movement sciences. Bernstein (1967) suggested that dexterity involves the continuous re-organisation of functional adaptive behaviours through exploratory learning. Bernstein (1967, pp. 228) conceptualized dexterity as "the ability to find a motor solution for any external situation, that is, to adequately solve any emerging motor problem correctly (i.e., adequately and accurately), quickly (with respect to both decision making and achieving a correct result), rationally (i.e., expediently and economically), and resourcefully (i.e., quick-wittedly and initiatively)" (italics in the original).

\section{The importance of Athlete Intrinsic Dynamics}

The fundamentality of an individual's dexterous interactions with a specific performance environment in sport has clear implications for understanding what each individual athlete can contribute to functioning in a specific performance environment. Whilst we have proposed that there is no role for an entity termed innate talent, we have previously highlighted the importance of intrinsic dynamics in athletes as complex adaptive systems (Araújo \& Davids, 2011). Kelso (1995) referred to intrinsic system dynamics as dispositional tendencies, propensities, capacities, and abilities that differ amongst individuals. The foundational capacities supported by an individual's intrinsic dynamics can be more or less functionally effective for stabilizing successful performance behaviours. An individual's intrinsic dynamics underpin self-regulation in sport performance, supporting the physical, cognitive, perceptual, psychological and emotional, and social interactions that emerge during performance (Davids et al., 2017). Intrinsic dynamics of complex adaptive systems are aligned with effectivities, referred to in ecological psychology as capacities for utilising affordances (opportunities for action), in specific performance domains. When an athlete's intrinsic dynamics cooperate with task dynamics in specific domains, then performance functionality will be enhanced (but goal achievement is still not guaranteed). Competing intrinsic and task dynamics can lead to misaligned relations between an athlete and a sport, providing a barrier to success at the highest levels (Seifert et al., 2016). Some tendencies can, of course, be transferrable in the sense of satisfying non-specific domain performance demands. It is important to note that this conceptualisation of transferability of dispositional tendencies in physical, cognitive, emotional and social interactions are harmonious with key ideas of a practitioner-led model of skill acquisition and talent development in high performance sport: The Athletics Skills Model (Wormhoudt et al., 2018). The ASM motto is 'first the athlete, then the specialist' promoting the view that, while the capacity to function at the highest performance levels may be domain-specific, the self-regulating nature of athlete functioning is deeply underpinned by non-domain specific capacities and evolving dispositional tendencies which may be psych-social, physical, and emotional.

In this way, ecological dynamics captures how some individuals may be predisposed over time towards functioning more effectively and efficiently in some sports environments than 
others. This approach does not preclude attempts to predict which athletes may have a greater potential to perform at the highest levels, the aim being to identify athletes with dispositional tendencies, in varied dimensions, to function effectively in specific performance contexts (over years and decades: the macro-timescale of sport development). The problem is not in the practice, but in the rationale for talent as innate.

A fruitful approach to meeting this challenge may be to design tasks which can help to assess and develop an individual's effectivities over time (de-prioritising current performance levels) and which may underpin future performance in specific sports. Clarification of specific tasks constraints of a competitive performance context that need to be satisfied, as well as their variations, (task dynamics) is needed in future research (Woods et al., 2016). However, it is important to heed evidence that analytics of sport performance at one specific point in time early in an athlete's career do not necessarily correspond to future performance at a senior level (Güllich, 2018).

\section{Summary}

We have proposed that talent, conceived as some individual innate entity that can predict exceptional future performance in young athletes, is an inadequate concept for sport science research and practice, i.e., more in the realm of myth and supposition than in scientific reality. Aligned with the approach advocated in the second key message of Baker and Wattie it is worth asking: What may be the main practical implications of an ecological dynamics conceptualisation of talent, skill and expertise in sport as increasingly functional adaptation and enhanced self-regulation of an individual in a specific performance environment? First, an important role seems to exist for identifying underlying tendencies, dispositions and capacities related to successful performance in specific domains which may signal future potential for individual functionality. In discerning athlete potential, the experiential knowledge of elite practitioners needs to be integrated with empirical knowledge over the extended macro-timescale of years and decades in high performance sports organisations (see Woods et al., 2016). Second, due to multiple dimensions of, and pathways, to successful athlete functioning in sport (Araújo \& Davids, 2010; Phillips et al., 2010), new models of coaching and athlete development are needed which integrate the work of specialist practitioners (Renshaw, Davids, Newcombe \& Roberts, 2019). Finally, these new models of athlete learning and preparation in the micro-structure of practice signal the need for a better balance between early specialisation in specific sport domains and the exploitation and development of general, transferable, functional tendencies which can be harnessed later over the macrotimescale of talent development (Wormhoudt et al., 2018).

\section{Funding}

DA was partially funded by the Fundação para a Ciência e Tecnologia, under Grant UID/DTP/ UI447/2019 to CIPER-Centro Interdisciplinar para o Estudo da Performance Humana (unit 447).

\section{Competing Interests}

The author(s) has/have declared that no competing interests exist.

\section{Data Availability Statement}

All relevant data are within the paper.

\section{References}

Araújo, D. \& Davids, K. (2011). Talent Development: From Possessing Gifts, to Functional Environmental Interactions. Talent Development and Excellence 3, 23-26.

Bernstein, N. A. (1967). The co-ordination and regulation of movements. London, England: Pergamon Press.

Davids, K. \& Araújo, D. \& (2010). The concept of 'Organismic Asymmetry' in sport science. Journal of Science and Medicine in Sport 13, 633-640.

Davids, K., Güllich, A., Araújo, D. \& Shuttleworth, R. (2017). Understanding environmental and task constraints on athlete development: Analysis of micro-structure of practice and macro-structure of development histories. In Routledge Handbook of Talent Identification and Development in Sport (Edited by J. Baker, S. Cobley, J. Schorer \& N. Wattie), pp.192206. Routledge: London.

Güllich, A. (2018). Sport-specific and non-specific practice of strong and weak responders in junior and senior elite athletics - A matched-pairs analysis. Journal of Sports Sciences, DOI: 10.1080/02640414.2018.1449089

Kelso JAS (1995). Dynamic Patterns: The Self-Organization of Brain and Behavior. Cambridge, MA: MIT Press

Phillips, E., Davids, K., Renshaw, I., \& Portus, M. (2010). Expert Performance in Sport and the Dynamics of Talent Development. Sports Medicine 40, 271-283.

Renshaw, I., Davids, K., Newcombe, D. \& Roberts, W. (2019). The Constraints-Led Approach: Principles for Sports Coaching and Practice Design (1st Ed.). Routledge Studies in Constraints-Based Methodologies in Sport. London: Routledge.

Seifert, L., Wattebled, L., Orth, D., L'Hermette, M., Boulanger, J. \& Davids, K. (2016). Skill transfer specificity shapes perception and action under varying environmental constraints. Human Movement Science 48, 132-141. 
Woods, C.T., McKeown, I., Haff, G.G. \& Robertson, S. (2016). Comparison of athletic movement between elite junior and senior Australian football players. Journal of Sports Sciences, 34, 1260-1265, DOI: 10.1080/02640414.2015.1107185

Wormhoudt, R., Savelsbergh, G.J.P., Teunissen, J.W. \& Davids, K. (2018). The Athletics Skills Model: Optimizing talent development through movement education. Routledge: London. 